\title{
PSP: rapid identification of orthologous coding genes under positive selection across multiple closely related prokaryotic genomes
}

Fei Su ${ }^{1,2}$, Hong-Yu Ou ${ }^{1,2}$, Fei Tao ${ }^{1,2}$, Hongzhi Tang ${ }^{1,2}$ and Ping $\mathrm{Xu}^{1,2^{*}}$

\begin{abstract}
Background: With genomic sequences of many closely related bacterial strains made available by deep sequencing, it is now possible to investigate trends in prokaryotic microevolution. Positive selection is a sub-process of microevolution, in which a particular mutation is favored, causing the allele frequency to continuously shift in one direction. Wide scanning of prokaryotic genomes has shown that positive selection at the molecular level is much more frequent than expected. Genes with significant positive selection may play key roles in bacterial adaption to different environmental pressures. However, selection pressure analyses are computationally intensive and awkward to configure.

Results: Here we describe an open access web server, which is designated as PSP (Positive Selection analysis for Prokaryotic genomes) for performing evolutionary analysis on orthologous coding genes, specially designed for rapid comparison of dozens of closely related prokaryotic genomes. Remarkably, PSP facilitates functional exploration at the multiple levels by assignments and enrichments of KO, GO or COG terms. To illustrate this user-friendly tool, we analyzed Escherichia coli and Bacillus cereus genomes and found that several genes, which play key roles in human infection and antibiotic resistance, show significant evidence of positive selection. PSP is freely available to all users without any login requirement at: http://db-mml.sjtu.edu.cn/PSP/.
\end{abstract}

Conclusions: PSP ultimately allows researchers to do genome-scale analysis for evolutionary selection across multiple prokaryotic genomes rapidly and easily, and identify the genes undergoing positive selection, which may play key roles in the interactions of host-pathogen and/or environmental adaptation.

Keywords: Orthologous genes, Positive selection, Synonymous and nonsynonymous substitutions, Bacterial microevolution, Bacillus cereus, Escherichia coli

\section{Background}

With the next-generation sequencing data "tsunami" in our midst, sets of closely related prokaryotic genomes suitable for comparative evolutionary studies have been available [1]. To date, well established cases of gene selection have been rare [2]. Big data mining of bacterial genomes has shown that positive selection is more widespread at the molecular level than expected under a restrictive interpretation of the neutral theory [3]. Genome-wide molecular selection analyses, designed to assess selection pressure across the entire genomes of different strains, have

\footnotetext{
* Correspondence: pingxu@sjtu.edu.cn

${ }^{1}$ State Key Laboratory of Microbial Metabolism, Shanghai Jiao Tong University, Shanghai 200240, P.R. China

${ }^{2}$ School of Life Sciences \& Biotechnology, Shanghai Jiao Tong University, Shanghai 200240, P.R. China
}

attempted to address the role of gene selection in the process of microevolution $[4,5]$, especially in host-pathogen interactions, and metabolic adaptation to a changing environment (stress, antibiotic). Positive selection studies on model bacteria, such as the species Escherichia coli $[4,6]$ and Listeria [7], or the genera Streptococcus [8,9] and Campylobacter [10] have revealed that positive selection is an essential part of natural selection to fix advantageous mutations, and improves the adaptability of bacteria in a wide range of environmental conditions.

A number of methods have been proposed for detecting positive selection in DNA or protein sequences [2]. The most common approach is to integrate evolutionary features into codon-based models, and to use probabilitybased theory to estimate the ratio $(\omega)$ of nonsynonymous $\left(d_{N}\right)$ and synonymous $\left(d_{S}\right)$ substitutions, such as 
implemented in the PAML [11] and FitModel [12]. Estimating the ratio $\omega$ gives a measure of selective pressure, indicating neutral evolution $(\omega=1)$, purifying selection $(\omega<1)$ and positive selection $(\omega>1)$. In the model of neutral evolution, the likelihood that a nonsynonymous mutation would go to fixation is the same as that for a synonymous mutation. Purifying selection can result in stabilizing selection through the purging of deleterious variations that arise. Positive selection pressure serves to maintain a given set of adaptive traits that aids in survival.

Several nice tools are currently available, such as IDEA [13], JCoDA [14] and WSPMaker [15]. However, they are not set up specifically to examine prokaryotic genomes, and they exhibit two major deficiencies: (i) the evolution selection analysis could be difficult to configure on a local computer for most biologists who are not familiar with phylogenetic or evolutionary theory, and (ii) excessively long computing times for analyzing several genomes at once are prohibitive. In this study, we present an open access web server called PSP (Positive Selection analysis for Prokaryotic genomes) to identify orthologous coding genes under positive selection across closely related prokaryotic genomes. It provides several core functions for in-depth analysis of evolutionary selection: retrieving the orthologous groups, generating codon-delimited and un-gapped alignments, removing recombination, building phylogenetic trees, and estimating $\omega$ under different models used by PAML/FitModel. Remarkably, PSP is able to facilitate efficient exploration of the identified orthologous genes at the metabolic pathway level by assignments and enrichments of KO (KEGG Orthology), GO (Gene Ontology) or COG (Clusters of Orthologous Groups) terms. Results are presented in a user-friendly web interface, which provides an efficient visualization of positive selection pressure on each orthologous groups.

\section{Implementation}

The PSP server first employs the BLAST-based orthologous genes assignment tools to build orthologous groups across multiple prokaryotic genomes being compared. Then it uses the codon-based strategy, similar to that of Petersen et al. [6], for identification of orthologous coding genes under positive selection. Finally, PSP facilitates functional exploration of the identified orthologous genes under positive selection by KEGG pathway mapping and enrichment of $\mathrm{KO}, \mathrm{GO}$ or COG terms. Here, we briefly describe PSP as an integrated framework of PAML, FitModel and several in-house programs as follows (Figure 1).

\section{Rapid identification of orthologous groups across multiple prokaryotic genomes}

The identification of orthologs is an important problem in the field of phylogenetic analyses. Basically, there are three types of relationships between orthologous genes, one-to-one, one-to-many and many-to-many [16]. In the study of Adrian et al. [17], OrthoMCL shows a balanced performance, such as the accuracy, the number of genomes analyzed and usability of the web-interface. Therefore, PSP integrates OrthoMCL to quickly identify the many-to-many orthologous relationship. PSP allows users to select or upload complete sequences and annotation details of closely related genomes for comparison. Users can also simultaneously upload thousands of annotated proteincoding genes as Multi-Fasta formatted files. With the default settings, OrthoMCL recognizes co-ortholog relationships with a BLASTp $E$-value cutoff of $1 \mathrm{e}-5$ and a minimum of $50 \%$ coverage. Then PSP performs homolog grouping using the Markov Cluster algorithm with an inflation value of 1.5. Interestingly, PSP runs on a high-performance server and can accept up to thirty comparator bacterial genomes simultaneously. Moreover, one-to-one orthologous relationship of genes could be identified using reciprocal BLAST best hit, which is the first and most widely used method for automatically establishing orthologous relationships [18].

\section{Optimization of multiple sequence alignment for automated phylogenetic analysis}

The protein-coding genes of the individual orthologous groups can be aligned by using MUSCLE or MAFFT. In connection with the automated phylogenetic analysis, PSP improves the coding sequence alignments by using the two following processes: (i) generation of codon-delimited alignments with ad hoc Perl scripts; (ii) maximization of the un-gapped alignment area by using MaxAlign to remove non-homologous sequences [19].

\section{Removal of gene recombination off orthologous groups}

Recombination (or gene conversion) is 10-50 times more likely to cause changes in nucleotide sequence than mutation [20]. To eliminate the influence of horizontal relationships in the positive selection detection, PSP is able to identify recombination signals among the aligned nucleotide sequences of orthologous groups. PSP performs a statistical test to identify recombination breakpoints by using GeneConv [21]. It pre-defines the $g$-scale parameter as 1 , which allows mismatches within a recombining fragment, and the inner fragment $P$-values with 10,000 random permutations. In addition, PSP also detects recombination with three other statistical tests implemented with the PhiPack package with default settings, including pairwise homoplasy index (PHI), Max $\chi^{2}$ and neighbor similarity score (NSS) [22]. Finally, if a recombination signal is detected to be significant by all four tests ( $P$-value $<0.05$ in each method), the alignment would be splitted into two or more fragments. 


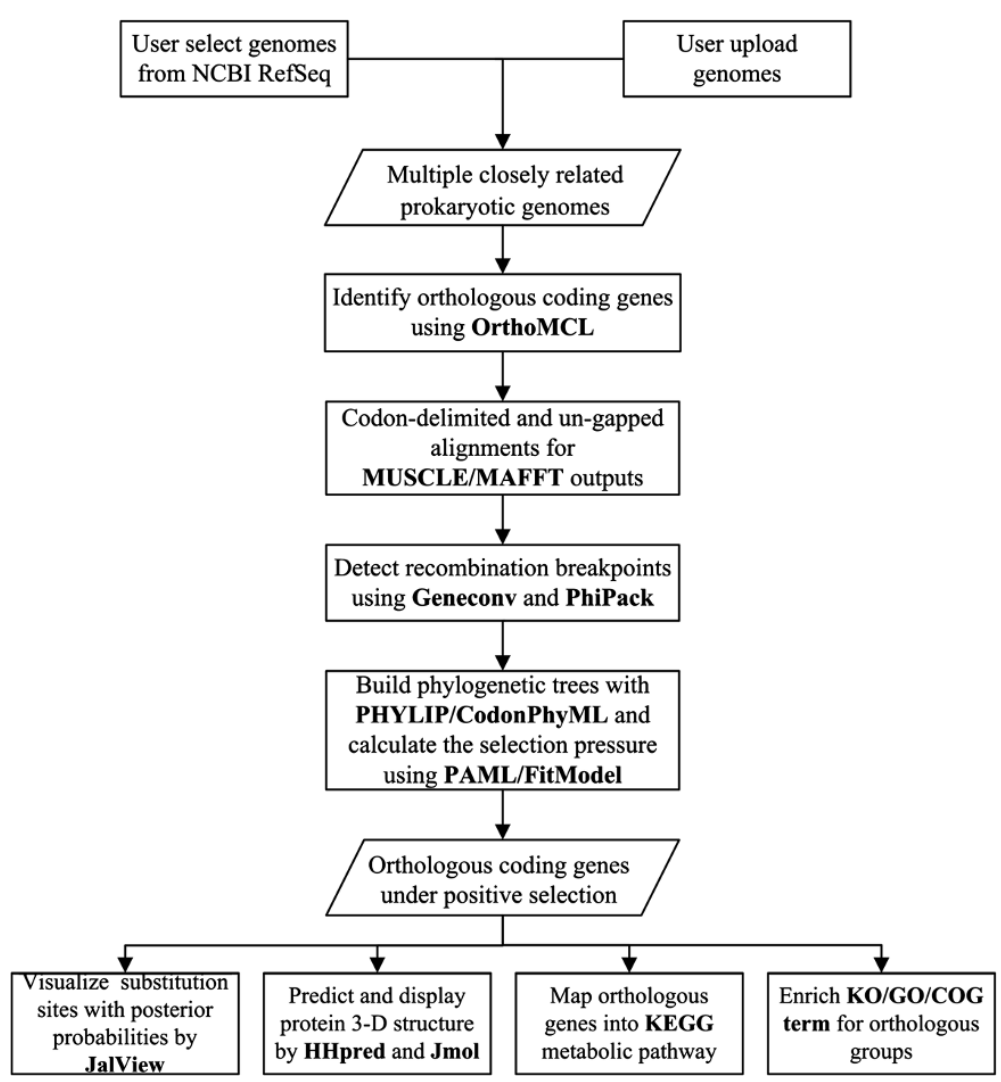

Figure 1 The strategy used by PSP to calculate prokaryotic selection pressure across multiple closely related genomes.

Detection of orthologous genes under positive selection Phylogenetic trees are built by the PHYLIP using maximum parsimony or neighbor-joining method. The trees are also able to be generated with Markov codon models by using CodonPhyML [23]. The evolutionary selection is subsequently implemented in the program PAML or FitModel. Only orthologous groups with enough data (at least 4 protein-coding genes) are used as input to detect positive selection, due to the poor quality of Bayes predictions based on small samples [24]. Because the lack of any methods to deal with alignment gaps properly in both programs, a cutoff of the percentages of sequence have data in PSP is used to filter the alignments column by column. In the PSP server, PAML uses three evolutionary models proposed by Yang et al. [25] (Additional file 1: Table S1): site model, strain-specific branch model and strain-specific site-branch model. In the strain-specific analysis, the branches of selected target strains are specified and referred to as "foreground branches" and the rest as "background branches", which is a powerful tool to detect the selection pressure during the process of environmental adaptation [4]. The in silico detection of evolutionary selection is computationally intensive, particularly using Bayes empirical Bayes to determine posterior probabilities (PP). PSP, which runs on a high-performance server, is able to rapidly calculate the $d_{N} / d_{S}$ ratios and screen orthologous coding genes under positive selection. Similarly, PSP also can apply switching Markov modulated codon models as implemented in the program FitModel to orthologous coding genes to accurately estimate the strength of selection. To most biologists who are not familiar with phylogenetic or evolutionary theory, PSP pre-defines the evolutionary models as described in Additional file 1: Table S1. PSP is also very flexible to set most key parameters in the PAML/FitModel and run strain-specific analysis freely for the evolutionary researchers. For each pair hypothesis, nested models are calculated by comparing the difference in log likelihood values to a $\chi^{2}$ statistic (LRT) for the detection of positive selection. If there is significant evidence for positive selection of any fragment, the orthologous genes, from which the fragment was separated, are suggested to be under positive selection. Notably, PSP also provides a user-friendly visualization tool for performing evolutionary analysis on orthologous coding genes. The embedded Java applet JalView [26] reports the PP values for all sites, which is helpful for users to determine nucleotide substitution at synonymous and nonsynonymous sites within protein-coding regions. Three-dimensional structural models of the protein of interest are predicted and displayed by HHpred [27] and 
Jmol [28]. Additionally, Primer3Plus [29] is integrated to facilitate design of PCR primers to assay orthologous genes based on similar selection among a panel of strains isolated from the same habitat.

\section{Functional investigation at metabolic pathway level}

To explore functions of the identified orthologous coding genes under positive selection, PSP performs KO mapping and GO/COG classification. First, to assign the KO terms, PSP uses the level of sequence identity and ratio of matching length to query length cut-off obtained from BLASTp. For each query against the locally installed KEGG gene database, the simple $H_{a}$-value homology score [30] is calculated as follows: $H_{a}=i \times\left(l_{m} / l_{q}\right)$, where $i$ is the level of identity between protein sequences in the region with the highest Bit score expressed as a ratio between 0 and 1 , $l_{m}$ the length of the highest scoring matching sequence (including gaps), and $l_{q}$ the query length. In this study, the $H_{a}$-value cutoff of 0.7 was used to assign $\mathrm{KO}$. PSP then enriches metabolic pathways with genes under positive selection by tracing back the hierarchical KO levels. The user can select one or more KEGG-archived closely related genomes (up to 20) as references. PSP calculates the $P$-value of each pathway category by using a hypergeometric distribution method [31]. In the same way, PSP provides enrichment analysis for COG functional terms based on RPSBLAST searches against the local CDD database, and GO slims analysis based on GOA database.

In the PSP pipeline, a large number of hypotheses are considered which could result in a high rate of Type-1 error even for a relatively stringent $P$-value cutoff. To reduce Type- 1 errors, PSP corrects the obtained $P$-values using the Q-value [32] to produce a $Q$-value at level of FDR $<0.2$.

\section{Results and discussion}

The PSP tool is applicable to a wide range of prokaryotic species. In this study, we applied PSP to do genome-wide positive selection analyses in two cases (Escherichia coli and Bacillus cereus) both to benchmark PSP and to illustrate its accuracy and usefulness for the exploration of data.

\section{Positive selection analysis of Escherichia coli}

We did a genome-wide positive selection analysis in four E. coli and two Shigella flexneri genomes (Additional file 1: Table S2), which were used by Petersen et al. [6]. The results of selection analysis in $E$. coli by Petersen et al. differ from those by Chen et al. [4] in several important ways, which only four genes were identified in both studies. To clarify the positive selection pressure of E. coli, we used six different models (M0, M1a, M2a, M3, $\mathrm{M} 2 \mathrm{a}+\mathrm{S} 1, \mathrm{M} 2 \mathrm{a}+\mathrm{S} 2$ ) to test for positive selection using PAML and FitModel. Additional file 1: Table S3 shows the $P$-values from LRTs obtained from four comparisons
(M0-M3, M1a-M2a, M2a-M2a + S1 and M2a + S1-M2a + S2) examined in this case. According to the idea of Yang (See detail from PAML FAQ), different evolutionary models always produce various parameter estimates and possibly different lists of site under positive selection. However, the sites under positive selection with posterior probabilities (PP > 99\%) in one model are more likely to detect under different models. The tests of M2a-M2a + S1 and M2a + $\mathrm{S} 1-\mathrm{M} 2 \mathrm{a}+\mathrm{S} 2$ could not access the points, which evolved under positive selection yet. Therefore, we compared the sites with PP $>99 \%$ across the remaining models $(11,562$ sites from model M2a and 16,479 from M3), in which about 9,105 (78.7\%) sites from M2a shared with the result of model M3 (55.2\%, Figure 2). In the previous studies, the M1a-M2a comparison also appears to be much more robust [33]. To ensure accuracy of the result, we used the results of M1a-M2a tests for further analysis. This analysis took about twenty hours to run the whole pipeline with M1a-M2a tests. During the analysis, we have identified 5,393 orthologous groups, among which 3,908 groups within at least 4 genes were analyzed by PSP (Table 1). Moreover, ninety-seven genes were detected to have significant signals for recombination by using both GenConv and PhiPack. GeneConv could also predict breakpoints where recombination has occurred. These breakpoints define fragments, which have different evolutionary histories due to recombination. Then, these orthologous genes were splitted into different fragments. In addition, about 117 genes were excluded due to MaxAlign, which was used to maximize the number of amino acid symbols that are present in gap-free columns. At last, we detected strong signals of positive selection pressure on 28 genes in E. coli $\mathrm{K} 12$, of which 13 genes were in the "core" groups (orthologous genes present in all genomes), while 15 genes were in the "dispensable" groups (orthologous genes

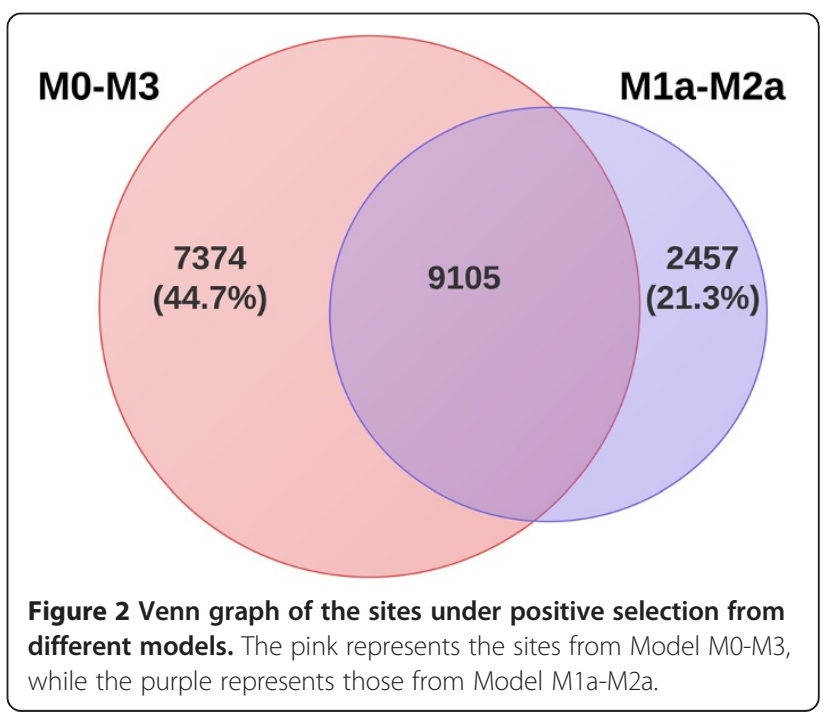


Table 1 Results of positive selection scanning during the analysis pipeline

\begin{tabular}{lccc}
\hline Results & This research & Petersen et al. & Chen et al. \\
\hline Num. of orthologous groups & 5,393 & - & - \\
Num. of orthologous (groups size $>=4)$ & 3,908 & 3,757 & 3,470 \\
Num. of genes removed by MaxAlign & 117 & - & - \\
Method for detecting recombination & GenConv \& PhiPack & HyPhy & GeneConv \& Reticulate \\
Num. of recombination & 97 & M1a-M2a \& M7-M8 & M1a-M2a \& Branch-Site Model \\
Evolutionary model & M1a-M2a & 23 & 29 \\
Num. of genes under positive selection in E. coli K12 & 28 & & 243 \\
\hline
\end{tabular}

present in two or more genomes but not all). However, there are still some differences between the results of Petersen and ours. This is primarily because Petersen focused on their studies specific to $E$. coli K12 and disregarded the orthologous groups that contain no K12 gene. In contrast, the methods used here identified selection pressure that acts in either "core" groups or "dispensable" groups. We identified 13 additional "dispensable" orthologous groups under positive selection. What's more, the different strategy of tests for recombination also affected the result of positive selection analysis. To estimate the accuracy of PSP's, we compared the $d_{S}$ value with previous studies, that the $d_{S}$ value is fairly independent across different datasets [13]. We calculated a median $d_{S}$ of $88.7 \times 10^{-3}$ among $E$. coli strains with the M0 model. The value we obtained is consistent with previous estimates of $27.0-51.3 \times 10^{-3}$ ( $P$-value $<0.001$, Wilcoxon test) [34].

According to the result of Petersan, we found 6 genes that were under positive selection in both studies.
Meanwhile, our approach resulted in the identification of 13 additional genes (excluding the genes involved in phage), including several that are very important in human infection and antibiotic resistance (Table 2). The gene fliC, which shows strong evidence for positive selection, encodes flagellin and is one of the most important virulence factors of Uropathogenic Escherichia coli (UPEC) [35]. Motility causes ascension of UPEC from bladder into kidney and helps UPEC to efficiently colonize the urinary tract. The gene $d a c \mathrm{C}$, first identified in $B$. subtilis genomes, encodes a putative 491-residue protein with homology to low-molecular-weight penicillin-binding proteins (PBPs) [36]. Expression of $d a c C$ in E. coli showed that this gene encodes an approximately $59-\mathrm{kDa}$ membrane-associated PBP, which is highly toxic when overexpressed. The gene eutA, which encodes the reactivating factor for ethanolamine utilization, is under the strong selection pressure. And recent researches imply that the utilization of ethanolamine provides a useful source of carbon/nitrogen that

Table 2 List of additional genes under positive selection across the six Escherichia coli genomes using the M1a-M2a test

\begin{tabular}{|c|c|c|c|c|c|c|}
\hline Gene & $P$-value ${ }^{a}$ & $\mathrm{KO}^{\mathrm{b}}$ & COG & Pathway & Enrichment $^{c}$ & Function \\
\hline alas & 0.000 & K01872 & J & ko00970 & 0.3094 & Alanyl-tRNA synthetase \\
\hline $\operatorname{cob} U$ & 0.046 & K02231 & $\mathrm{H}$ & ko00860 & 0.6536 & $\begin{array}{l}\text { Bifunctional cobinamide kinase and } \\
\text { cobinamide phosphate guanylyltransferase }\end{array}$ \\
\hline dacC & 0.024 & K07258 & M & ko00550 & 0.6806 & D-alanyl-D-alanine carboxypeptidase \\
\hline eutA & 0.000 & K04019 & $\mathrm{E}$ & ko00564 & 0.6025 & Reactivating factor for ethanolamine ammonia lyase \\
\hline flic & 0.000 & K02406 & $\mathrm{N}$ & ko02020 & 0.1939 & Flagellar filament structural protein \\
\hline hchA & 0.000 & K05523 & $\mathrm{R}$ & - & - & Hsp31 molecular chaperone \\
\hline$n f s A$ & 0.000 & K07734 & K & - & - & Nitroreductase A \\
\hline$p g l$ & 0.000 & K07404 & G & ko00030 & 0.3210 & 6-phosphogluconolactonase \\
\hline yadB & 0.000 & K01894 & J & - & - & Glutamyl-Q tRNA synthetase \\
\hline yebN & 0.000 & - & $\mathrm{S}$ & - & - & Conserved inner membrane protein \\
\hline$y d d V$ & 0.000 & K13069 & $\mathrm{T}$ & - & - & Predicted diguanylate cyclase \\
\hline$y f d F$ & 0.041 & - & - & - & - & Hypothetical protein \\
\hline$y g f l$ & 0.013 & - & K & - & - & Putative DNA-binding transcriptional regulator \\
\hline ygeR & 0.000 & K12943 & M & - & - & Lipoprotein \\
\hline
\end{tabular}

a The $P$-value shown is the smallest one among different fragments if there is a strong signal of recombination.

b "_" indicates that no functional term was assigned.

"The "enrichment" represents $P$-value of hypergeometric distribution for each KEGG pathway. 
promotes successful colonization of the intestine [37]. Studies have also demonstrated that mutation in the $n f s \mathrm{~A}$ is associated with nitrofuran resistance, because it encodes cellular reductase that can reduce members of the nitrofuran family [38]. hch A and $y d d \mathrm{~V}$ both are the key genes involved in resistance of a wide range of stress, such as heat and antibiotic [39,40]. The other genes, yebN (conserved inner membrane protein) and ygeR (lipoprotein), have no clear function. But properties predicted from their sequence suggest that they encode the proteins locating on the membrane surface. They may possibly serve as receptors for phages or antibiotics.

Genes under positive selection in Bacillus cereus genomes A genome-wide molecular selection scanning using PSP was also done for Bacillus cereus group (twenty-eight completely sequenced strains of $B$. cereus, $B$. thuringiensis and $B$. anthracis listed in the Additional file 1: Table S4).
PSP compared all the 159,473 annotated genes in the 28 Bacillus genomes with maximum likelihood and strain-specific branch-site model using $B$. anthracis as "foreground branches" in about two hundred hours. Totally, thirty-two groups showed evidences for positive selection after correcting for multiple tests, of which 14 groups were the "core" genes while 18 groups were the "dispensable". As in previous studies $[4,6]$, we found that proteins that typically undergo positive selection are involved in interaction with the phages or infections (Table 3). For example, alkyl hydroperoxide reductase is an enzyme for protection strains from peroxide-induced stress and plays key roles not only in colonization but also in potential virulence [41]. In addition, a number of genes also show evidence for positive selection with the connection to antibiotic resistance, mass transport system and transcriptional regulation (Figure 3).

Table 3 Genes that show evidences for positive selection in B. anthracis group

\begin{tabular}{|c|c|c|c|c|}
\hline Gene $^{a}$ & $P$-value ${ }^{b}$ & $\mathrm{KO}^{\mathrm{c}}$ & COG $^{\mathrm{C}}$ & Function \\
\hline$a m p C$ & 0.000 & K01467 & $\mathrm{V}$ & $\beta$-lactamase \\
\hline ubiE & 0.000 & - & $\mathrm{H}$ & SAM-dependent methyltransferase \\
\hline rluA & 0.000 & - & J & Ribosomal large subunit pseudouridylate synthase $D$ \\
\hline$g / p F$ & 0.000 & - & G & Glycerol uptake facilitator protein \\
\hline$f l g \mathrm{~L}$ & 0.000 & - & N & Flagellin \\
\hline pbp2 & 0.000 & - & M & Penicillin-binding protein \\
\hline paiB & 0.000 & K07734 & K & Protease synthase and sporulation negative regulatory protein \\
\hline araj & 0.000 & - & G & $A B C$ transporter \\
\hline baes & 0.000 & - & $\mathrm{T}$ & Sensor histidine kinase \\
\hline$a f u B$ & 0.000 & K02011 & $\mathrm{P}$ & Iron (III) transport system permease protein \\
\hline ptr2 & 0.000 & - & $E$ & Multidrug resistance protein \\
\hline$a h p F$ & 0.000 & K03387 & O & Alkyl hydroperoxide reductase, subunit $F$ \\
\hline COG3708 & 0.000 & - & $\mathrm{S}$ & Transcriptional regulator, AraC family \\
\hline gcd 1 & 0.000 & K00966 & MJ & GMPP; mannose-1-phosphate guanylyltransferase \\
\hline COG4653 & 0.000 & - & $\mathrm{R}$ & Putative prophage LambdaBa04, major capsid protein \\
\hline rsuA & 0.000 & K06183 & J & RNA pseudouridine synthase family protein \\
\hline purR & 0.000 & - & K & Sugar-binding transcriptional regulator, Lacl family \\
\hline CW/A & 0.000 & K01447 & M & $\mathrm{N}$-acetylmuramoyl-L-alanine amidase \\
\hline$n t r B$ & 0.000 & K00936 & $\mathrm{T}$ & Sporulation kinase \\
\hline COG1079 & 0.000 & - & $\mathrm{R}$ & ABC transporter, permease \\
\hline$r n j$ & 0.000 & K12574 & $\mathrm{R}$ & RNA-metabolising metallo-beta-lactamase \\
\hline COG3127 & 0.000 & - & Q & ABC transporter, permease \\
\hline COG1979 & 0.000 & K00100 & C & Alcohol dehydrogenase \\
\hline fepD & 0.000 & K02015 & $P$ & Iron complex transport system permease protein \\
\hline nupC & 0.000 & K03317 & $\mathrm{F}$ & Nucleoside permease NupC \\
\hline
\end{tabular}

More details are available at http://202.120.45.186/ webserver/kaks/detail.php?jobld=UrK5YPUZE1.

a Only the genes, which have clear function, were listed in this table.

$\mathrm{b}$ The $P$-value shown is the smallest one among different fragments if there is a strong signal of recombination.

c "_" indicates that no functional term was assigned. 


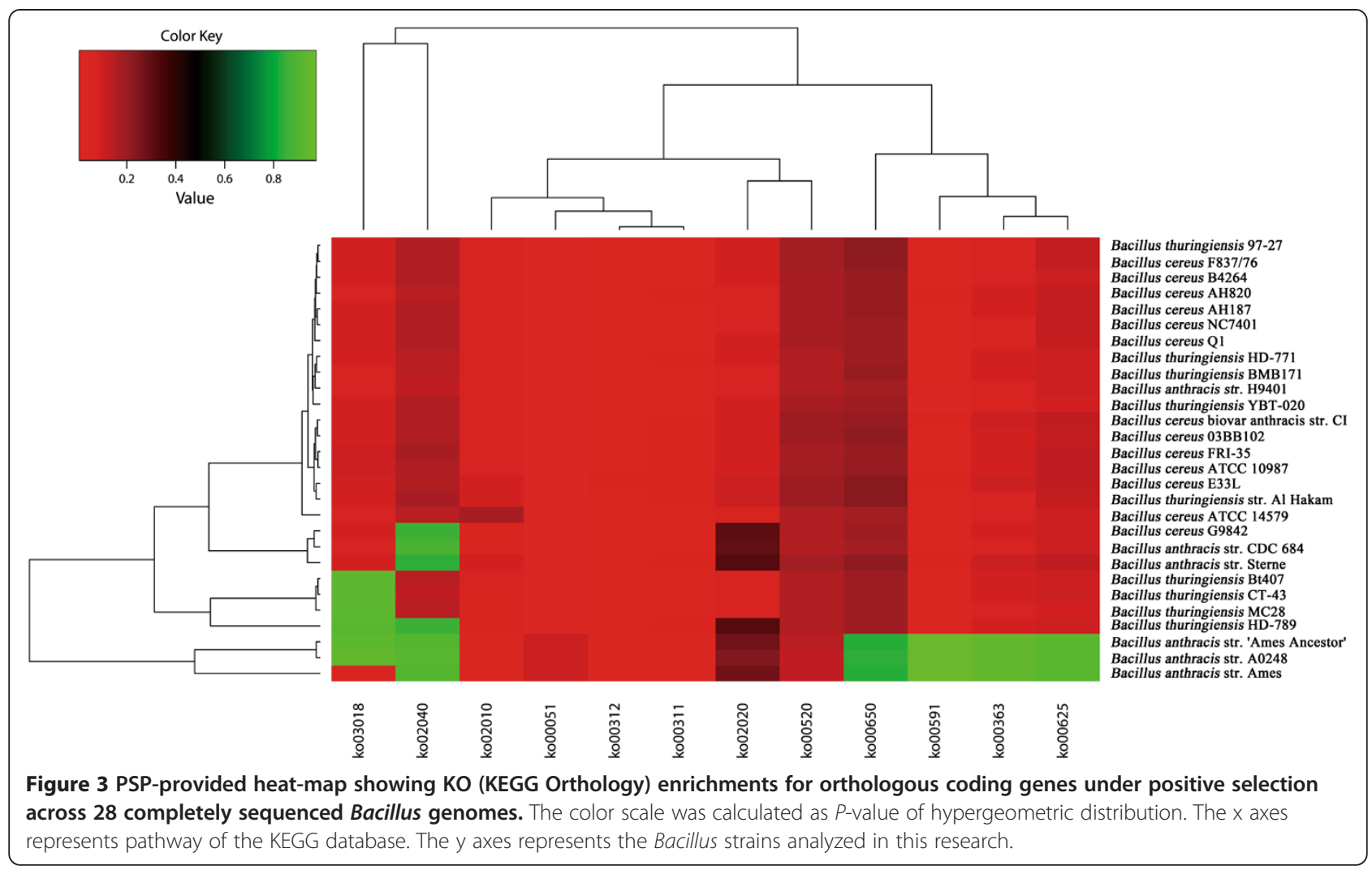

Antibiotic is well recognized to impose strong selective pressure. Especially the proteins on the surface of cell, which are more likely to be exposed, are preferential target for antibiotic-resistance-related selective pressure. In this case, we identified a $\beta$-lactamase with strong positive selection $(a m p C, P$-value $<0.001)$, which could provide resistance to $\beta$-lactam antibiotics like penicillins and cephamycins. We built the phylogenetic tree of $a m p C$ genes from all analyzed Bacillus strains (Figure 4), in which all from $B$. anthracis are clustered together. However, the gene from $B$. anthracis str. CDC 684 shows significant difference from the others and was removed by MaxAlign. Susceptibility to $\beta$-lactam-containing compounds is a common trait of $B$. anthracis, because gene expression of $\beta$-lactamase is usually not sufficient to provide resistance to the agents [42]. Therefore, $\beta$-lactam agents have been used worldwide to treat anthrax, which would speed up the evolution of genes and aid bacteria populations in becoming resistant to antibiotics. And at the same time, $p h p 2$, which is a type of PBPs, is under strong positive selection. The strong selection pressure is attributable to the process of "arms race" between antibiotic and pathogen. With the protein structures of AmpC and Php2, the majority of sites predicted to be under positive selection are surface exposed (Figure 5). Positive selection in the Php2 seems to be associated with selection to avoid recognition by the antibiotic.
Mass transport system is another effective way for microorganism to resist antibiotic, although it is probable that they may have other natural physiological functions [43]. There are seven genes involved in the mass transport system, which shows the significant enrichment compared to the references $(P$-value $=0.0395$ in the strain Ames Ancestor). The araJ gene was regarded as nonessential membrane protein of unknown function. Recently, it is believed to belong to a large class of multidrug resistance translocators and in particular to the major facilitator superfamily [44]. The orthologous genes of ptr2, which encode a peptide transporter, present in all B. cereus genomes and are regarded as a multidrug resistance protein. In this research, we found it is under strong positive selection and may have important roles to extrude drugs. Iron acquisition genes are also important contributors to $B$. anthracis, while iron limitation is a component of host defense against infection [45]. The gene fepD, which shows strong evidence for positive selection, is a virulence-associated gene and involved in iron-chelating $\mathrm{ABC}$ uptake systems [46]. The gene afuB was reported to differentially express upon treatment with antibiotic, and is also very important for iron transporter [47]. Porins are also important in interaction with the host immune system and could work as receptors for phages or antibiotics [6]. Aquaglyceroporin $g l p \mathrm{~F}$ selectively conducts the passage of small hydrophilic across the inner membrane of $B$. cereus 


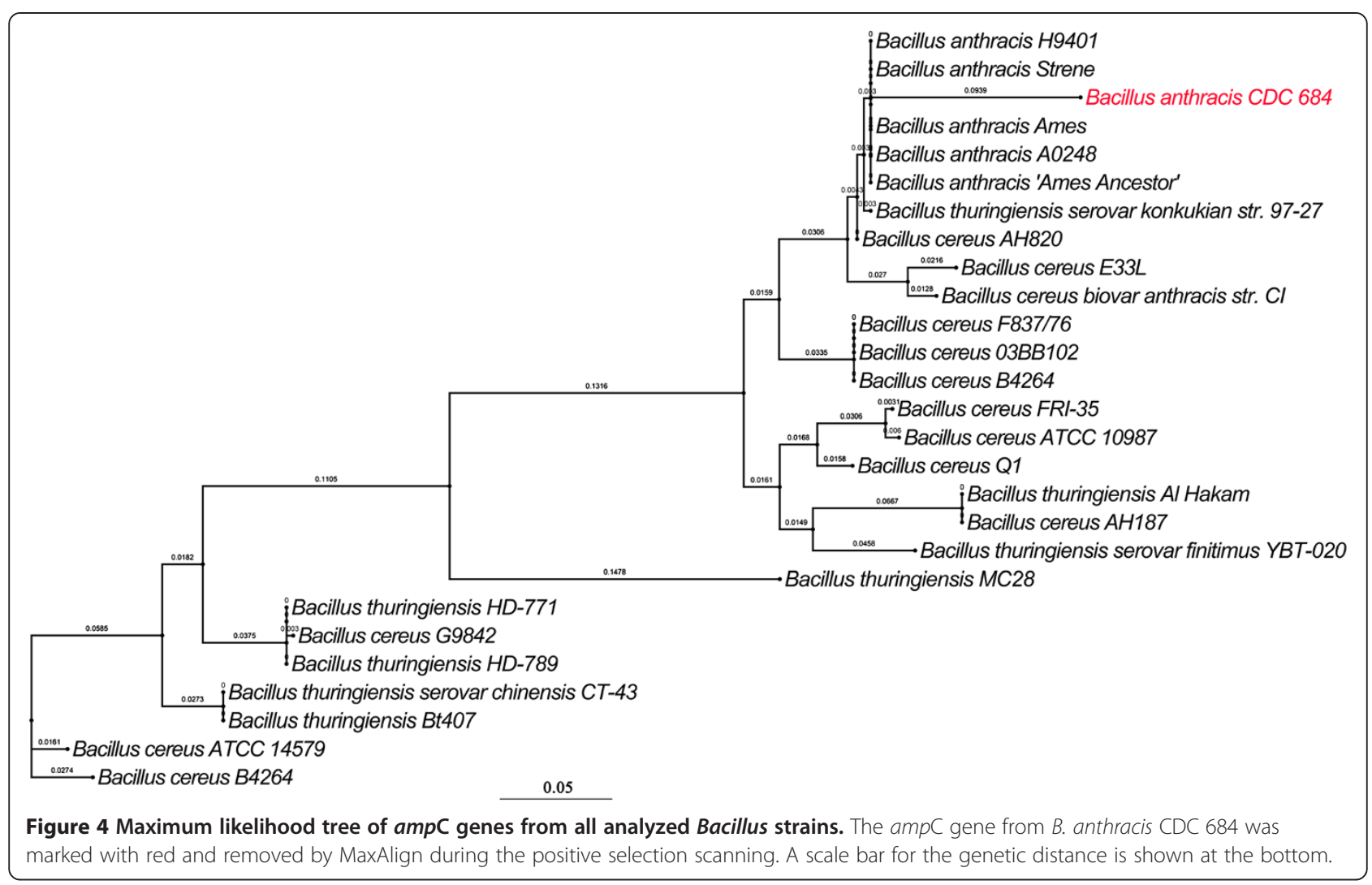

[48]. The function of remaining two putative $A B C$ transporters is still unknown, but one of them (COG3127) was predicted to involve in lysophospholipase L1 biosynthesis, which may be a good potential target for new antibiotics.

We also identified two transcriptional repressors (purR and $\operatorname{araC}$ ) and a two-component system (ntrB-ntrC), which show strong evidences for positive selection. Autoregulation of purR controls the expression of many genes involved in purine biosynthetic pathway in B. subtilis [49]. $n t r B$, a member of the ntrB- ntrC two-component system, encodes the signal-transducing kinase/phosphatase nitrogen regulator, on the regulated phosphatase activity involved in nitrogen regulation. These genes are known to have multiple functions in different ways, which are likely that the positive selection from the interaction of hostpathogen simultaneously improve the adaptation of microorganisms by acting on the versatile proteins, such as metabolic adaptation.

\section{Conclusions}

The PSP web server, which integrates a wide variety of useful analytical and functional tools, has been developed to rapidly identify orthologous coding genes under positive selection across up to thirty user-selected or user-supplied

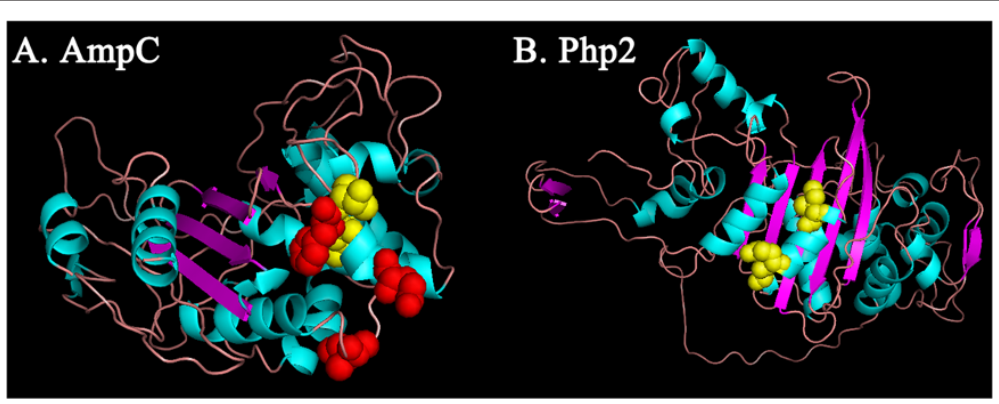

Figure 5 Three-dimensional structures of $\mathrm{AmpC}$ and Pbp2 proteins. A) Three-dimensional structure of $\beta$-lactamase (AmpC). B) Three-dimensional structure of penicillin-binding protein (Pbp2). Sites that show evidences for positive selection (PP > 99\%) are depicted as red spheres. And sites (PP $>95 \%)$ are depicted as yellow spheres. 
prokaryotic genomes. Hosted by a high-performance server and with easy navigation and flexible input options, we present it as a quick and comprehensive genome microevolution tool for biologists. Remarkably, PSP excludes the effect of gene recombination and incorporates functional investigation at the metabolic pathway level. In the future, we plan to improve the computing power of PSP markedly with Paralleled PAML. The upgraded version will be also able to map the positive selection sites to three-dimensional structures of proteins. We propose that a tool such as PSP will support genome-scale analysis for evolutionary selection, aimed at defining genomic biomarkers of evolutionary lineage, phenotype, pathotype, environmental adaptation and/or disease-association of diverse bacterial species.

\section{Availability and requirements}

Project name: PSP:Positive Selection analysis for Prokaryotic genomes.

Project home page: http://db-mml.sjtu.edu.cn/PSP/, or http://202.120.45.186/ webserver/kaks/.

Operating system(s): CentOS.

Programming language: PHP 5.3+, JavaScript, HTML5, MYSQL, PERL, R.

License: PSP is available free of charge to academic and non-profit institutions.

Any restrictions to use by non-academics: Please contact authors for commercial use.

\section{Additional file}

Additional file 1: Table S1. Evolutionary models provided by PSP.

Table S2. Six Escherichia coli genomes used in case one. Table S3.

$P$-values of 4-pair comparisons of genes which found under positive

selection by Petersen et al. Table S4. Twenty-eight completely

sequenced Bacillus cereus genomes under analysis in case two.

\section{Competing interests}

The authors declare that they have no competing interests.

\section{Authors' contributions}

FS designed and implemented the PSP project. FS and $\mathrm{HO}$ drafted the paper. HT and FT contributed important ideas and advices. PX supervised the project. All authors read and approved the final manuscript.

\section{Acknowledgments}

This work was supported, in part, by the grants from National Basic Research Program of China (2013CB733901) and from Chinese National Natural Science Foundation (31230002, 31170082, and 31121064).

Received: 24 July 2013 Accepted: 26 December 2013 Published: 27 December 2013

\section{References}

1. Novichkov PS, Ratnere I, Wolf YI, Koonin EV, Dubchak I: ATGC: a database of orthologous genes from closely related prokaryotic genomes and a research platform for microevolution of prokaryotes. Nucleic Acids Res 2009, 37:D448-D454.

2. Yang Z, Bielawski JP: Statistical methods for detecting molecular adaptation. Trends Ecol Evol 2000, 15:496-503.
3. Bustamante CD, Fledel-Alon A, Williamson S, Nielsen R, Hubisz MT, Glanowski S, Tanenbaum DM, White TJ, Sninsky JJ, Hernandez RD, et al: Natural selection on protein-coding genes in the human genome. Nature 2005, 437:1153-1157.

4. Chen SL, Hung CS, Xu J, Reigstad CS, Magrini V, Sabo A, Blasiar D, Bieri T, Meyer RR, Ozersky P, et al: Identification of genes subject to positive selection in uropathogenic strains of Escherichia coli: a comparative genomics approach. Proc Natl Acad Sci U S A 2006, 103:5977-5982.

5. Kosiol C, Anisimova M: Selection on the protein-coding genome. Methods Mol Biol 2012, 856:113-140.

6. Petersen L, Bollback JP, Dimmic M, Hubisz M, Nielsen R: Genes under positive selection in Escherichia coli. Genome Res 2007, 17:1336-1343.

7. Orsi RH, Sun Q, Wiedmann M: Genome-wide analyses reveal lineage specific contributions of positive selection and recombination to the evolution of Listeria monocytogenes. BMC Evol Biol 2008, 8:233.

8. Lefebure T, Stanhope MJ: Evolution of the core and pan-genome of Streptococcus: positive selection, recombination, and genome composition. Genome Biol 2007, 8:R71.

9. Suzuki H, Lefebure T, Hubisz MJ, Pavinski Bitar P, Lang P, Siepel A, Stanhope $\mathrm{MJ}$ : Comparative genomic analysis of the Streptococcus dysgalactiae species group: gene content, molecular adaptation, and promoter evolution. Genome Biol Evol 2011, 3:168-185.

10. Lefebure T, Stanhope MJ: Pervasive, genome-wide positive selection leading to functional divergence in the bacterial genus Campylobacter. Genome Res 2009, 19:1224-1232.

11. Yang Z, Nielsen R: Estimating synonymous and nonsynonymous substitution rates under realistic evolutionary models. Mol Biol Evol 2000, $17: 32-43$.

12. Guindon S, Rodrigo AG, Dyer KA, Huelsenbeck JP: Modeling the site-specific variation of selection patterns along lineages. Proc Natl Acad Sci U S A 2004, 101:12957-12962.

13. Egan A, Mahurkar A, Crabtree J, Badger JH, Carlton JM, Silva JC: IDEA: Interactive Display for Evolutionary Analyses. BMC Bioinformatics 2008, 9:524.

14. Steinway SN, Dannenfelser R, Laucius CD, Hayes JE, Nayak S: JCoDA: a tool for detecting evolutionary selection. BMC Bioinformatics 2010, 11:284

15. Lee YS, Kim TH, Kang TW, Chung WH, Shin GS: WSPMaker: a web tool for calculating selection pressure in proteins and domains using window-sliding. BMC Bioinformatics 2008, 9(Suppl 12):S13.

16. Tatusov RL, Galperin MY, Natale DA, Koonin EV: The COG database: a tool for genome-scale analysis of protein functions and evolution. Nucleic Acids Res 2000, 28:33-36.

17. Altenhoff AM, Dessimoz C: Phylogenetic and functional assessment of orthologs inference projects and methods. PLOS Comput Biol 2009, 5:e1000262

18. Gabaldon T: Large-scale assignment of orthology: back to phylogenetics? Genome Biol 2008, 9:235.

19. Gouveia-Oliveira R, Sackett PW, Pedersen AG: MaxAlign: maximizing usable data in an alignment. BMC Bioinformatics 2007, 8:312.

20. Feil EJ, Maiden MC, Achtman M, Spratt BG: The relative contributions of recombination and mutation to the divergence of clones of Neisseria meningitidis. Mol Biol Evol 1999, 16:1496-1502.

21. Sawyer SA: GENECONV: A computer package for the statistical detection of gene conversion. Distributed by the author, Department of Mathematics, Washington University in St Louis 1999, http://www.math.wustl.edu/ sawyer/ geneconv/.

22. Bruen T, Bruen T: PhiPack: PHI test and other tests of recombination. Montreal, Quebec: McGill University; 2005.

23. Gil M, Zanetti MS, Zoller S, Anisimova M: CodonPhyML: Fast Maximum Likelihood Phylogeny Estimation under Codon Substitution Models. Mol Biol Evol 2013, 30:1270-1280

24. Anisimova M, Bielawski JP, Yang Z: Accuracy and power of bayes prediction of amino acid sites under positive selection. Mol Biol Evol 2002, 19:950-958.

25. Yang Z, Wong WS, Nielsen R: Bayes empirical bayes inference of amino acid sites under positive selection. Mol Biol Evol 2005, 22:1107-1118.

26. Waterhouse AM, Procter JB, Martin DM, Clamp M, Barton GJ: Jalview Version 2-a multiple sequence alignment editor and analysis workbench. Bioinformatics 2009, 25:1189-1191.

27. Soding J: Protein homology detection by HMM-HMM comparison. Bioinformatics 2005, 21:951-960. 
28. Herraez A: Biomolecules in the computer: Jmol to the rescue. Biochem Mol Biol Educ 2006, 34:255-261.

29. Untergasser A, Nijveen H, Rao X, Bisseling T, Geurts R, Leunissen JA: Primer3Plus, an enhanced web interface to Primer3. Nucleic Acids Res 2007, 35:W71-W74.

30. Shao Y, He X, Harrison EM, Tai C, Ou HY, Rajakumar K, Deng Z: mGenomeSubtractor: a web-based tool for parallel in silico subtractive hybridization analysis of multiple bacterial genomes. Nucleic Acids Res 2010, 38:W194-W200.

31. Tang $\mathrm{H}, \mathrm{Wu} \mathrm{Cl}$ : A new method for estimating nonsynonymous substitutions and its applications to detecting positive selection. Mol Biol Evol 2006, 23:372-379.

32. Storey JD, Tibshirani R: Statistical significance for genomewide studies. Proc Natl Acad Sci U S A 2003, 100:9440-9445.

33. Wong WS, Yang Z, Goldman N, Nielsen R: Accuracy and power of statistical methods for detecting adaptive evolution in protein coding sequences and for identifying positively selected sites. Genetics 2004, 168:1041-1051.

34. Jordan IK, Rogozin IB, Wolf YI, Koonin EV: Essential genes are more evolutionarily conserved than are nonessential genes in bacteria. Genome Res 2002, 12:962-968.

35. Rogers TJ, Paton JC, Wang H, Talbot UM, Paton AW: Reduced virulence of an fliC mutant of Shiga-toxigenic Escherichia coli O113:H21. Infect Immun 2006, 74:1962-1966.

36. Pedersen LB, Murray T, Popham DL, Setlow P: Characterization of dacC, which encodes a new low-molecular-weight penicillin-binding protein in Bacillus subtilis. J Bacteriol 1998, 180:4967-4973.

37. Garsin DA: Ethanolamine utilization in bacterial pathogens: roles and regulation. Nature Rev Microbiol 2010, 8:290-295.

38. Paterson ES, Boucher SE, Lambert IB: Regulation of the nfsA Gene in Escherichia coli by SoxS. J Bacteriol 2002, 184:51-58.

39. Tagliabue L, Maciąg A, Antoniani D, Landini P: The yddV-dos operon controls biofilm formation through the regulation of genes encoding curli fibers' subunits in aerobically growing Escherichia coli. FEMS Immun Med Microbiol 2010, 59:477-484.

40. Mujacic M, Baneyx F: Regulation of Escherichia coli hchA, a stress-inducible gene encoding molecular chaperone Hsp31. Mol Microbiol 2006, 60:1576-1589.

41. Cosgrove K, Coutts G, Jonsson IM, Tarkowski A, Kokai-Kun JF, Mond JJ, Foster $\mathrm{SJ}$ : Catalase (KatA) and alkyl hydroperoxide reductase (AhpC) have compensatory roles in peroxide stress resistance and are required for survival, persistence, and nasal colonization in Staphylococcus aureus. J Bacteriol 2007, 189:1025-1035.

42. Chen Y, Succi J, Tenover FC, Koehler TM: Beta-lactamase genes of the penicillinsusceptible Bacillus anthracis Sterne strain. J Bacterio/ 2003, 185:823-830.

43. Allen HK, Donato J, Wang HH, Cloud-Hansen KA, Davies J, Handelsman J: Call of the wild: antibiotic resistance genes in natural environments. Nature Rev Microbiol 2010, 8:251-259.

44. Edgar R, Bibi E: MdfA, an Escherichia coli multidrug resistance protein with an extraordinarily broad spectrum of drug recognition. J Bacteriol 1997, 179:2274-2280.

45. Maresso AW, Chapa TJ, Schneewind O: Surface protein IsdC and Sortase B are required for heme-iron scavenging of Bacillus anthracis. J Bacteriol 2006, 188:8145-8152.

46. Bhagat N, Virdi JS: Distribution of virulence-associated genes in Yersinia enterocolitica biovar $1 \mathrm{~A}$ correlates with clonal groups and not the source of isolation. FEMS Microbiol Lett 2007, 266:177-183.

47. Melnikow E, Schoenfeld C, Spehr V, Warrass R, Gunkel N, Duszenko M, Selzer PM, Ullrich HJ: A compendium of antibiotic-induced transcription profiles reveals broad regulation of Pasteurella multocida virulence genes. Vet Microbiol 2008, 131:277-292.

48. Beijer L, Nilsson RP, Holmberg C, Rutberg L: The glpP and glpF genes of the glycerol regulon in Bacillus subtilis. J Gen Microbiol 1993, 139:349-359.

49. Saxild HH, Brunstedt $\mathrm{K}$, Nielsen Kl, Jarmer H, Nygaard P: Definition of the Bacillus subtilis PurR operator using genetic and bioinformatic tools and expansion of the PurR regulon with glyA, guaC, pbuG, xpt-pbuX, yqhZ-folD, and pbuO. J Bacteriol 2001, 183:6175-6183.

doi:10.1186/1471-2164-14-924

Cite this article as: Su et al:: PSP: rapid identification of orthologous coding genes under positive selection across multiple closely related prokaryotic genomes. BMC Genomics 2013 14:924.

\section{Submit your next manuscript to BioMed Central and take full advantage of:}

- Convenient online submission

- Thorough peer review

- No space constraints or color figure charges

- Immediate publication on acceptance

- Inclusion in PubMed, CAS, Scopus and Google Scholar

- Research which is freely available for redistribution 